\title{
Communication Strategies of New Media for Women Leaders-taking Lani-Rob Ray, Philippine Vice President as An Example
}

\author{
Qian Liu ${ }^{1}$, Jingwen $\mathrm{Gao}^{2}$, Xing $\mathrm{Lu}^{3}$, and Yilong Chen ${ }^{4^{*}}$ \\ ${ }^{1}$ Jinan University, International College, Canton, Guangzhou 510632; \\ ${ }^{2}$ Jinan University, School of Journalism and communication, Canton, Guangzhou 510632; \\ ${ }^{3}$ Jinan University, Physics College, Canton, Guangzhou 51063; \\ ${ }^{4}$ Jinan University, Physical Education College, Canton, Guangzhou 510632 \\ *The corresponding author
}

\begin{abstract}
The influence of network news and social internet is surging on the background of new media through which Women leaders interact with netizes, consequently strategies on new media attract the attention of researchers. Having sort out relevant theories, we have analyzed online new media of Lani-Rob Ray, Philippine Vice President, and the main three accounts for three network. Review has made on topics and events from January to October, 2016 such as presidential election and China South Seas Arbitration Issue. We discover that under new media several methods have impacts on pubic domestically and internationally, taking advantages of new media worldly to express their statements objectively and prudently enables it possible to actively communicate with netizes, so the kindness and regulation abilities will be given full play. In the end, it provides new ways for china's female leaders on communication strategies of new media to learn.
\end{abstract}

Keywords: New media; Social media; Women leaders; Network news; Communication strategy

\section{女性领导人新媒体的沟通策略--以菲律宾副总统}

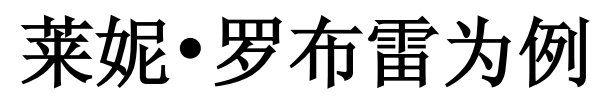

\author{
刘倩 ${ }^{1}$ ，高敬文 ${ }^{2}$ ，陆星 ${ }^{3}$ ，陈义龙 ${ }^{4 *}$
}

(1. 暨南大学 国际学院，广东广州 510632；2.暨南大学 新闻与传播学院，广东 广州 510632;

3. 暨南大学 物理学院, 广东广州 510632; 4. 暨南大学 体育学院, 广东 广州 510632)

摘要: 新媒体背景下, 网络新闻和社交网络影响力剧增。女性领导人通过新媒体进行与网民沟通, 相关的新媒体策略也 是研究的重点。在对相关理论进行了梳理后, 对菲律宾副总统莱妮・罗布雷的网络新闻媒体, 以及社交网络上三个主要账号 进行分析。对 2016 年 1 月至 10 月以来的涉及的话题和重要事件, 例如：大选以及南海仲裁等事件进行了回顾。发现女性领 导人在新媒体背景下，通过多种方式对本国和外国民众进行互动和影响，客观谨慎表达了政见，借助新媒体平台在全球范围 内, 可以积极与网民产生互动，充分发挥亲和力和协调能力。为中国的女性领导人新媒体的沟通策略提供了借鉴的新思路。

关键词: 新媒体; 社交媒体; 女性领导人; 网络新闻; 沟通策略

中图分类号：G 206.2 文献标志码：A

引言

新媒体影响力剧增的今天，国际政坛中，女性领导人起到了越来越重要的作用，也通过新媒体平台产 生了重要影响。国际上, 从欧洲的英国的伊丽莎白二世到中国的宋庆龄、吴仪等, 都在国际政坛上产生了 重要影响。新媒体平台作为重要的途径, 为国内外的女性领导人进行沟通, 起到了越来越重要的作用, 而 
其中亦有很多消极影响是需要在政府决策中避免的。针对这些策略的研究是重要而宜乏的。新媒体平台日 益体现其重要性, 政府也通过新媒体平台扩大影响力。甚至有学者研究认为通过社交网络可以开展外交或 者影响政府决策。但前人研究聚焦在新媒体平台或者针对女性政治家的研究很多，而对女性政治家社交媒 体和网络新闻的研究却相对有限。本研究选取的研究对象是菲律宾副总统莱妮・罗布雷 Leni Robredo, 作 为和中国有一定渊源的女性领导人，她的新媒体沟通策略能给中国带来一定启示。

本文在对相关理论进行了梳理后, 通过对莱妮网络新闻媒体以及社交网络上三个主要账号进行整理, 对自 2016 年 1 月至 10 月以来的数据进行分析。研究了女性领导人在新媒体背景下, 如何通过多种策略, 对本国和外国民众进行影响和开展互动, 为社交网络和网络新闻等的新媒体渠道使用, 提供了借鉴的新思 路。

\section{1 文献综述}

\section{1 女性领导人的研究崛起}

随着越来越多的女性领导人步入政坛，这些女性领导人起着不可忽视的作用。

针对女政治家的研究很多，从女权主义，或者心理学，或者传播学角度的都有。学者万明认为，女性 领导人在国际政治上有区别于传统男性政治的特征和意义，显现出柔性化特征。[1]而媒体就是她们重要 的渠道。有大量的关于女性领导的成长路径, 职务晋升 [2], 媒体使用的研究。有部分研究表明媒体很多 时候起到的是消极作用, 学者鞠红的研究认为媒体的作用十分消极, 不利于女性领导人的形象塑造。[3] 这是女性领导人在媒体传播中需要避免的。一个很好的案例就朴槿惠, 有学者认为其学历能力, 机会经历 和女性特质助推她成功上位。[4]但是在媒体的狂轰滥炸之下，其个人形象禁毁。

\section{2 新媒体上网络新闻和社交媒体的研究丰富}

一方面, 新媒体平台日益体现其重要性。Ellison, N. B., Lampe, C., \& Steinfield, C 对社交网络 脸书 286 个样本的数据进行回归分析后, 发现社交网络的使用有利于心理健康, 能够提高自信和达到自我 满足感。[5]可见对网民来说, 新媒体平台日益突显其重要性。

另一方面, 政府也通过新媒体平台扩大影响力。政务微博, 网络政务新闻发布是重要的应用方向, 学 者 Antoniadis, K., Vrana, V., \& Zafiropoulos, C. 就做过政务 twitter 账号的研究。[6]中国学者王娟, \& 王钰. 对中国政务微博也进行了研究, 并对政务微博的具体维护技巧提出了建议。[7]可见, 政府也充分 利用新媒体平台发声, 扩大影响力。

亦有学者研究认为通过社交网络可以进行外交。传媒外交属公共外交 [8], 赵启正认为, 除了政府与 政府之间的外交活动之外， “政府与民众、民众与民众之间的信息流动关系都成为了公共外交的主要内 容。”[9] 也有学者认为传媒外交可以对外交施加影响。[10]西方学者博萨 - 埃布认为媒体外交指的是 “运用媒体阐述和推进对外政策”。[11] 其操作性的定义包括互联网的社交媒体平台及发布的信息 [12]。所以本文关注社交网络的推文和网络新闻。

\section{3 案例选择莱妮・罗布雷的介绍}

本文的案例是菲律宾副总统莱妮・罗布雷 Leni Robredo。其亲属中有祖籍福建的, 和中国有一定渊源, 对中菲关系上到底起到了重要的作用, 她的新媒体沟通策略会给中国带来一定启示, 而针对她的研究却比 较有限。 


\section{2 方法和数据说明}

本文数据包括网络新闻中相关的报道和社交网络上的数据。搜集方法是通过爬虫对互联网上包含相应 关键词的新闻进行搜索, 或者对指定平台帐号信息进行查询。通过 facebook 社交网站信息的收集和分析, 对莱妮・罗布雷进行观察。分析的样本来自 2016 年 1 月 1 日到 10 月 31 日，对以下三个账号的脸书账号 内容做为抓取对象, 包括 1085 条博文和 403865 条一级评论。

表 1: 三个账号详情分析（截至 2016 年 11 月 1 日）

\begin{tabular}{|c|c|c|c|c|c|}
\hline \multirow[t]{3}{*}{ 账号名 } & 用户 & 主页 & \multirow[t]{3}{*}{ 内容 } & \multirow[t]{3}{*}{ 属性 } & \multirow[t]{3}{*}{ 网址 } \\
\hline & 在讨 & 总赞 & & & \\
\hline & 论 & 数 & & & \\
\hline LeniRob & 122,5 & 10899 & 活动, 主张, 照片, & 副总统个人官 & https://www. faceboo \\
\hline redoPH & 13 & 49 & 视频 & 方账号 & k. com/LeniRobredoPH \\
\hline VPPilip & 10,15 & 14,13 & 活动, 宣传, 主张, & 副总统办公室 & https://www. faceboo \\
\hline inas & 2 & 5 & 照片, 视频 & 官方账号 & k. com/VPPilipinas \\
\hline YouthFo & 36 & 23,70 & 组织信仰, 号召, 宣 & 菲律宾青年领 & https://www. faceboo \\
\hline rLeni & & 3 & 传, 学生相关内容 & 袖团体账号 & k. com/YouthForLeni \\
\hline
\end{tabular}

\section{3 基于社交网络和网络新闻分析}

\section{1 新媒体平台涉及的主要话题}

在网络新闻分析中, 对莱妮 - 罗布雷姓名、中菲关系、选举、南海仲裁案等关键词, 搜索了英文的相 关新闻报道, 并抽取了比较有代表性的 12 篇新闻报道和 5 条中国相关的网络新闻作分析。发现主要议题 涉及: 菲律宾总统选举, 南海仲裁案以及涉中报道。

通过网络新闻地分析, 发现副总统莱妮 - 罗布雷支持本国政府在仲裁案的表现。在美欧与菲律宾关系 方面, 莱妮 - 罗布雷比较亲欧美, 重视欧美的经济援助。且在她的近期的一些新闻报道中表面呈现了与总 统的矛盾, 但实则体现出协调作用, 支持有弹性外交。在提及与中国相关议题时, 持保守态度, 例如转发 言辞比较极端的 Riyoh 的视频而避免直接评论; 推文也包括与中国相关组织和部门的一些会面, 例如 2016 年 7 月 5 日的博文是和中华和平发展协会的面见; 另外还有一些和中国相关报道, 例如福建庆祝副总统当 选, 举行游行的新闻等等。

可见网络新闻，主要的工作是稳定内部民心，对海外民众表达政治主张，莱妮则通过网络新闻，体现 了女性领导人的亲和力以及协调能力，保持国家对外有弹性的外交政策。

\section{2 不同社交网络渠道的分工沟通}

莱妮社交网络账三个账号分工明确，内容也不拘泥于文字，图像、音视频均有涉及，可谓多角度、广 视听、分层次进行多媒体内容打造。 


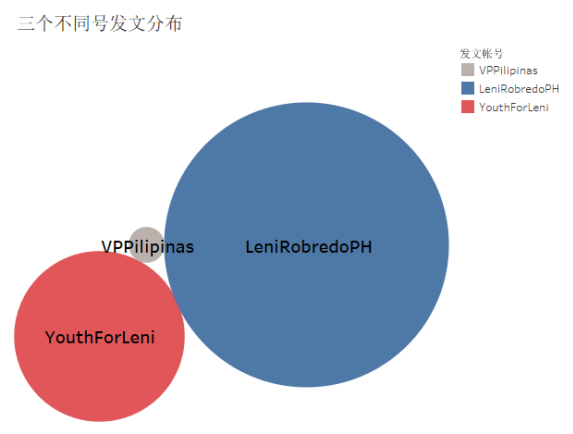

图 1 三个不同帐号发文数量分布

从图 1 可以看出三个不同帐号发文数量分布情况。发文最多的是个人官方账号 LeniRobredoPH，这个 账号, 主要内容发布的是个人的活动、主张、照片、视频等。副总统办公室官方账号 VPPilipinas 是新的 官方账号, 其用户的提及和主页的点赞都少了一个数量级, 内容也和 LeniRobredoPH 有部分重复, 主要是 官方的宣传、活动、主张等内容。形式也是文字、照片、图片、视频兼顾, 但在活跃度和影响力上, 仍然 需要进一步圈粉。YouthForLeni 的主要限制于团体内部圈子的讨论, 主要是在广播信息和号召行动。

可见莱妮 - 罗布雷的三个帐号有分工与合作, 在选举期间比较活跃, 主动发文和互动评论都与选举活 动有一定相关性, 充分利用了新媒体平台上的社交媒体进行了与网民的互动特别是青年支持者的发动。

\section{3 网民互动的分析}

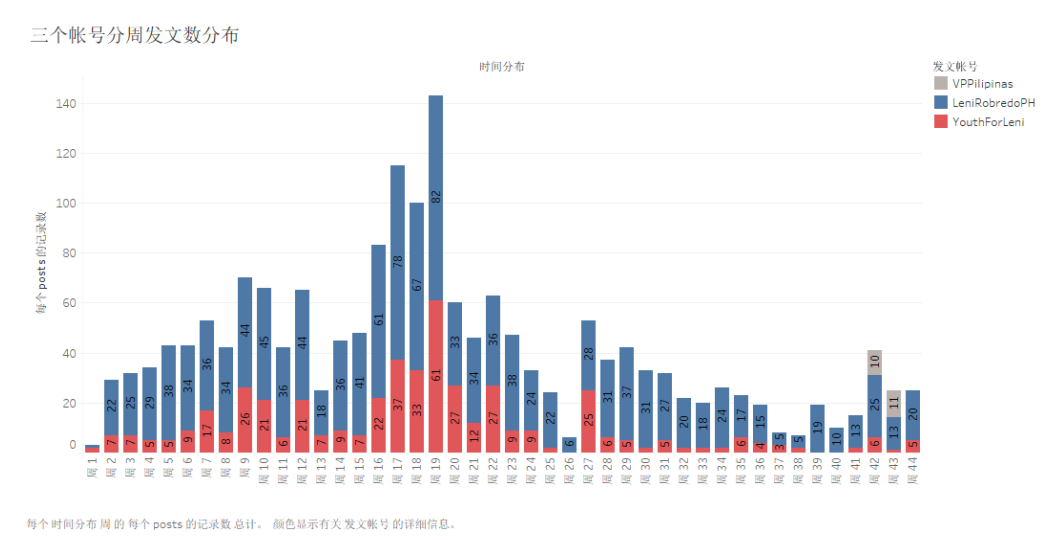

图 2 三帐号发文数按周柱状图分布

从图 2 看出随着发文的数量的增加, 评论数也有相应的提高, 特别是对于 LeniRobredoPH 帐号, 这种 联动效应比较明显, 影响力比较大。进一步分析具体发文和评论可知: LeniRobredoPH 有一批铁粉, 在这 三个 facebook 账号的互动中, 通过数据统计, 发现超过 100 次互动留言的铁粉数量有 127 人。

具体分析信息传播的特性是以事件驱动发文, 带动互动。以 2016 大选事件为例, 三个帐号主要的博 文和评论数目都集中在选举前后 3 个月, 自年初开始预热后, 发布博文数大量增加, 博文带动评论, 大约 从第 13 周, 即 4 月上旬开始急速上升, 到第 17 周, 即 5 月初到达顶峰, 在其后维持了近一个月的热度, 之后开始慢慢折返式回落, 在第 36 周, 即 9 月时大体回复到选举前状况。其中 YouthForLeni 账号是青年 支持者账号，5 月底到达顶峰，在其后开始回落。 


\section{4 结论}

通过以上分析，可以发现莱妮新媒体传播策略包括以下几点:

\section{1 发布信息重视青年及海外受众}

合理分工, 通过多种形式主张, 照片, 视频等, 抓住不同受众的心, 特别是青年和海外受众。其 YouthForLeni 帐号宣传其主张, 倡导透明度, 问责制和参与政府, 是青年支持者的聚合地。通过与母校 互动, 发动校友力量, 在新媒体平台培养青年粉丝。英语使用十分普遍, 这为通过网络平台传播政见, 开 启传媒外交渠道打通了重要的语言关卡, 网罗了大量本土用户和海外的用户。

通过新媒体平台开展传媒外交, 需要重视不同平台, 多种内容配合分工, 且需尊重青年用户的习惯和 重视英文作为国际通用语言的地位。

\section{2 传播信息体现女性亲和力及协调能力}

发挥女性领导人优势, 主要体现在圈粉体现亲合力以及政见表达中的协调力体现两方面。

莱妮在与网民互动中体现出相当的亲和力, 特别是青年和女性支持者中。莱妮有相当的女性支持者: 例如: 网民 “米莲米莲” 在一则灾后访问的博文后留言: “The best VP I' ve seen so far since i started voting! I' m so proud to have a woman VP. ”莱妮 - 罗布雷配合总统, 体现柔和有弹性的外交政策, 在政见表达中, 有相当的协调能力。以女性的立场、沟通方式和灵活协调的手段, 来配合强势权力政治的 不足, 更具包容性。女性领导人在新媒体平台上开展传媒外交, 如何发挥优势体现亲和力和协调能力, 莱 妮起到了很好的示范作用。

\section{3 客观谨慎表达政见}

作为国家领导人, 莱妮有着中国祖籍的身份渊源, 在政见中特别谨慎关于中国的态度, 除了较少提及, 也多数通过客观的报道和转发相关言论以及新闻评论等方式传递对中国态度。

莱妮通过这些新媒体的沟通策略, 在不同平台上, 与青年用户和海外用户互动, 客观谨慎表达政见, 借助新媒体渠道在全球范围内与网民产生了互动, 充分发挥了女性领导人亲和力和协调能力。这些新媒体 平台上传播策略对中国女性领导人有一定借鉴意义的。

\section{5 致谢}

本文受广东省自然科学项目 “大数据背景下的社会與情监测研判体系与智能决策研究”, 以及教育部 人文社会科学青年基金项目 “品牌国际化联结策略的构成及机制研究” (16YJC630037) 支持。数据收集 受到暨南大学與情与社会管理研究中心支持。

\section{参考文献:}

[1] 万明. 女性视角下的国际政治沟通理念与实践——女性领导人为例 [J]. 国际观察，2013，6：004.

[2] 陈怀珍. 女性领导的成长路径及职务晋升研究——我国省部级女性常委为例 $[\mathrm{J}]$. 宁夏大学学报: 人文社会科学 版, 2013，35(6): 105-108.

[3] 鞠红. 性别视角下的媒体与女性领导人一以美国三任女国务卿为例 [J]. 新闻传播, 2011 (4)：47-48.

[4] 杨彩利. 女性领导人成长的一个标本一以扑槿惠为例 [J]. 领导科学, 2014 (6)：48-49.

[5] Ellison N B, Steinfield C, Lampe C. The benefits of Facebook “friends:” Social capital and college students' use of online social network sites[J]. Journal of Computer - Mediated Communication, 2007, 12 (4): 1143-1168. 
[6] Antoniadis K, Zafiropoulos K, Vrana V. A Method for Assessing the Performance of e-Government Twitter Accounts[J]. Future Internet, 2016, 8(2): 12.

[7] 王娟, 王钰. 中国政务微博发展初探 [J]. 采. 写. 编, 2012 (4)：9-11.

[8] 赵可金. 媒体外交及其运作机制 $[J]$. 世界经济与政治, 2004（4）：21-26.

[9] 赵启正. 公共外交与跨文化交流[M]. 中国人民大学出版社, 2011.

[10］孙建平，谢奇峰. “传媒外交” 初探 [J]. 现代传播：北京广播学院学报，2002（3)：70-72.

[11] Gilboa E. Mass communication and diplomacy: A theoretical framework[J]. Communication Theory, 2000, $10(3): 275-309$.

[12］陆佳怡. 媒体外交：一种传播学视角的解读 $[J]$. 国际新闻界，2015，37 (4)：92-105.

\section{References:}

[1] Wan Ming. Communication Theory and Practice of International Politics from Women Perspective-Take Female Leaders as Example [J].International Review, 2013, 6:004.

[2] Chen Huaizhen. Study on Growth Path and Promotion of Women's Leadership-Take Standing Committee of Provincial Women in China as Example [J].Journal of Ningxia University (Social Science Edition), 2013, 35 (6):105-108.

[3] Ju Hong. Media and Women Leaders from Gender Perspective-Take Three US Female Secretary as Example [J].Journalism Communication, 2011 (4):47-48.

[4] Yang Caili. A Specimen of Female Leaders Growth-take Park Geun-hye as example [J].Leadership Science, 2014 (6):48-49.

[5] Ellison N B, Steinfield C, Lampe C. The benefits of Facebook "friends:" Social capital and college students' use of online social network sites [J]. Journal of Computer - Mediated Communication, 2007, 12(4): 1143-1168.

[6] Antoniadis K, Zafiropoulos K, Vrana V. A Method for Assessing the Performance of e-Government Twitter Accounts [J]. Future Internet, 2016, 8(2): 12.

[7] Wang Juan, Wang Yu. Primary Exploration of Development of Micro-blogging in Chinese Government [J].Journalism Probe, 2012 (4):9-11.

[8] Zhao Kejin. Media Diplomacy and Its Operating Mechanism [J].World Economics and Politics, 2004 (4):21-26.

[9] Zhao Qizheng. Public Diplomacy and Intercultural Communication [M].China Renmin University Press, 2011.

[10] Sun Jianping, Xie Qifeng. Primary Exploration of "Media Diplomacy" [J].Modern Communication: Journal of Beijing Broadcasting Institute, 2002(3):70-72.

[11] Gilboa E. Mass communication and diplomacy: A theoretical framework [J]. Communication Theory, 2000, 10(3): 275-309.

[12]Lu Jiayi. Media Diplomacy: Interpretation of an Aspect of Communication [J].Chinese Journal of Journalism \& Communication, 2015, 37 (4):92-105. 